\title{
Hydrogen-Bonding-Assisted Brønsted Acid and Gold Catalysis: Access to Both (E)- and (Z)-1,2-Haloalkenes via Hydrochlorination of Haloalkynes
}

\author{
Xiaojun Zeng, ${ }^{\dagger}$ Shiwen Liu, ${ }^{\dagger}$ Gerald B. Hammond, ${ }^{*}, \star \odot$ and Bo $\mathrm{Xu}^{*}{ }^{\dagger}$
}

${ }^{\dagger}$ College of Chemistry, Chemical Engineering and Biotechnology, Donghua University, 2999 North Renmin Lu, Shanghai 201620, China

${ }^{\ddagger}$ Department of Chemistry, University of Louisville, Louisville, Kentucky 40292, United States

\section{Supporting Information}

ABSTRACT: We have developed an efficient synthesis of both $(Z)$ - and (E)-chlorohaloalkenes via hydrochlorination of haloalkynes, based on two distinct hydrogen-bond-networkassisted catalytic systems: Brønsted acid catalysis and gold catalysis. Both systems offer high stereoselectivity, good chemical yields, and diverse functional group tolerance.

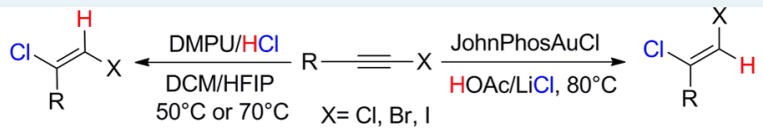

hydrogen bond assisted acid catalysis

syn-addition

13 examples, up to $92 \%$ cationic gold catalysis anti-addition

28 examples, up to $98 \%$

KEYWORDS: hydrogen bonding, gold catalysis, Brønsted acid catalysis, hydrochlorination, haloalkynes

\begin{abstract}
A lkenyl chlorides are important synthetic building blocks - that have been used extensively in cross-coupling reactions ${ }^{1}$ such as Stille couplings, ${ }^{2}$ Suzuki couplings, ${ }^{3}$ Sonogashira couplings, ${ }^{4}$ and Buchwald-Hartwig aminations. ${ }^{5}$ In addition, alkenyl chlorides are commonly found in biologically active natural products. ${ }^{6,7}$ More specifically, 1,2chlorohaloalkenes are especially important synthons due to presence of two synthetic handles. ${ }^{8}$ The possibility of consecutive transition-metal-catalyzed cross-coupling reactions permits stereocontrolled synthesis of highly substituted alkenes. ${ }^{8}$ For example, 1,2-chlorohaloalkenes have been exploited as key building blocks en route to natural products Pan-Bcl-2-Inhibitor ${ }^{9}$ and chartellines ${ }^{10}$ (Figure 1).
\end{abstract}
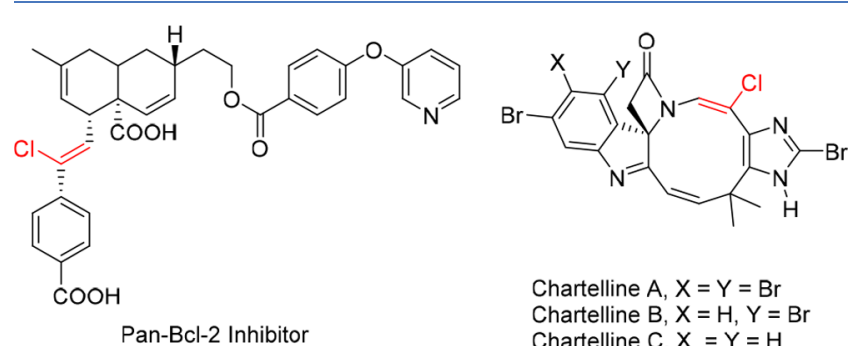

Chartelline $\mathrm{A}, \mathrm{X}=\mathrm{Y}=\mathrm{Br}$ Chartelline $\mathrm{B}, \mathrm{X}=\mathrm{H}, \mathrm{Y}=\mathrm{Br}$ Chartelline $\mathrm{C}, \mathrm{X}=\mathrm{Y}=\mathrm{H}$

Figure 1. Alkenyl chloride-containing natural products that can be prepared from 1,2-chlorohaloalkenes.

1,2-Chlorohaloalkenes can be prepared via directly halogenation of alkynes (Scheme 1a). ${ }^{11}$ These reactions usually give anti addition products via a halonium intermediate, but poor regio- and stereoselectivies restrict their usefulness. ${ }^{11}$ Given that the hydrochlorination of alkynes is a well-established method to prepare chloroalkenes, ${ }^{12}$ the hydrochlorination of haloalkynes should be a straightforward method to prepare 1,2-
Scheme 1. Existing Methods for the Synthesis of 1,2Chlorohaloalkenes

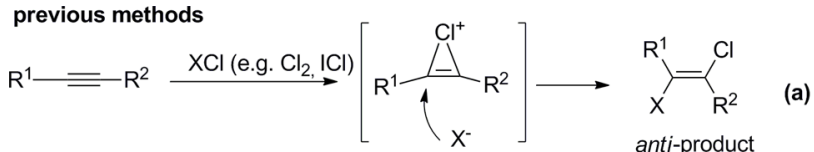

$$
\begin{aligned}
& \left.\mathrm{R} \underset{\mathrm{F}}{=} \frac{\mathrm{Pd}(\mathrm{OAC})_{2}, \mathrm{Cod}}{\mathrm{HOAC} / \mathrm{LiCl}, 80^{\circ} \mathrm{C}} \mathrm{Cl}\right|_{\mathrm{R}} ^{\mathrm{C}} \mathrm{X}=\mathrm{Cl} \text { or } \mathrm{Br} \\
& \mathrm{R} \underset{\mathrm{BF}_{4}^{-}}{=} \mathrm{I}^{+} \mathrm{Ph} \underset{\mathrm{or} \mathrm{LiX} / \mathrm{AcOH}}{\mathrm{HX} / \mathrm{MeOH}} \mathrm{R}_{\mathrm{R}}^{\mathrm{I}^{+} \mathrm{Ph}} \mathrm{X}^{-} \mathrm{Cl} \text { or } \mathrm{Br} \\
& \text { this work } \\
& \text { hydrogen bond assisted } \\
& \text { acid catalysis } \\
& \text { syn-addition } \\
& \text { cationic gold catalysis } \\
& \text { anti-addition }
\end{aligned}
$$

chlorohaloalkenes. ${ }^{13}$ Indeed, Zhu and co-workers ${ }^{14}$ described a clever route to $(Z)$-1,2-chlorohaloalkenes via Pd catalyzed antihydrochlorination of haloalkynes (Scheme $1 \mathrm{~b}$ ). Ochiai and coworkers reported a metal-free Michael type addition of halides to alkynyl(phenyl)iodonium tetrafluoroborates (Scheme 1c). ${ }^{11 \mathrm{~b}}$ However, these methods suffer from moderate chemical yields, and only provide access to anti-addition products. Herein we present two distinct hydrogen bond

Received: October 18, 2017

Revised: December 10, 2017

Published: December 15, 2017 
network assisted catalytic systems that offer selective access to both (E)- and (Z)-chlorohaloalkenes, based on Brønsted acid and gold catalysis ${ }^{15}$ (Scheme 1d).

We first tested our newly developed hydrochlorination reagent $\mathrm{DMPU} / \mathrm{HCl}$ on model substrate bromoalkyne 1a (Table 1). ${ }^{16}$ Although $\mathrm{HCl}$ is a strong acid, almost no reaction

\section{Table 1. Optimization of the Hydrochlorination of} Bromoalkyne $1 a^{a}$

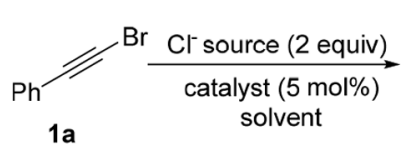

\begin{tabular}{|c|c|c|c|c|c|}
\hline no. & solvent & catalyst & $\mathrm{HCl}$ source & $\begin{array}{l}\text { temp } \\
\left({ }^{\circ} \mathrm{C}\right)\end{array}$ & $\begin{array}{l}\text { yield \% } \\
(\mathbf{2 a}: 3 \mathbf{a})\end{array}$ \\
\hline 1 & DCM & - & $\mathrm{HCl} / \mathrm{DMPU}$ & 50 & trace \\
\hline 2 & DCE & - & $\mathrm{HCl} / \mathrm{DMPU}$ & 70 & trace \\
\hline 3 & $\mathrm{PhCH}_{3}$ & - & $\mathrm{HCl} / \mathrm{DMPU}$ & 80 & trace \\
\hline 4 & $\begin{array}{c}\text { DCM/HFIP } \\
(3 / 1)\end{array}$ & - & $\mathrm{HCl} / \mathrm{DMPU}$ & 50 & $98(20: 1)$ \\
\hline 5 & $\begin{array}{c}\text { DCM/HFIP } \\
(3 / 1)\end{array}$ & - & $\mathrm{HCl} / \mathrm{Et}_{2} \mathrm{O}$ & 50 & $35(4: 1)$ \\
\hline 6 & $\begin{array}{c}\text { DCM/HFIP } \\
(3 / 1)\end{array}$ & - & $\mathrm{HCl} / i-\mathrm{PrOH}$ & 50 & $98(8: 1)$ \\
\hline 7 & $\begin{array}{c}\text { DCM/HFIP } \\
(3 / 1)\end{array}$ & - & $\mathrm{HCl} /$ dioxane & 50 & $97(11: 1)$ \\
\hline 8 & dioxane & $\mathrm{TiO}_{2} / \mathrm{Au}, 3 \mathrm{~A} \mathrm{MS}$ & $\mathrm{HCl} /$ dioxane & 90 & 0 \\
\hline 9 & DCM & $\begin{array}{l}\mathrm{CpRuCl}(\operatorname{cod}) / \\
\mathrm{PPh}_{3}\end{array}$ & $\mathrm{HCl} / \mathrm{DMPU}$ & 50 & $46(1: 0.6)$ \\
\hline 10 & DCM & JohnPhosAuCl & $\mathrm{HCl} / \mathrm{DMPU}$ & 50 & 10 \\
\hline 11 & DCE & JohnPhosAuCl & $\mathrm{HCl} / \mathrm{DMPU}$ & 80 & $37(1: 17)$ \\
\hline 12 & $\begin{array}{c}\text { DCM/HFIP } \\
(3 / 1)\end{array}$ & JohnPhosAuCl & $\mathrm{HCl} / \mathrm{DMPU}$ & 50 & $98(1: 0.5)$ \\
\hline 13 & HOAc & JohnPhosAuCl & $\mathbf{L i C l}^{b}$ & 80 & $\begin{array}{l}97 \\
\quad(<1 / 99)\end{array}$ \\
\hline 14 & DCE & JohnPhosAuCl & $\mathrm{TMSCl}^{b} / \mathrm{H}_{2} \mathrm{O}$ & 80 & $50(1 / 49)$ \\
\hline 15 & DCE & JohnPhosAuCl & $\mathrm{AcCl}^{b} / \mathrm{H}_{2} \mathrm{O}$ & 80 & $71(1 / 8)$ \\
\hline
\end{tabular}

${ }^{a}$ Conditions: 1a $(0.2 \mathrm{mmol}), \mathrm{HCl}$ source $(0.4 \mathrm{mmol}), \mathrm{DCM} / \mathrm{HFIP}$ $(3: 1,0.4 \mathrm{~mL}), 8$ h. ${ }^{b} 5$ equiv.

took place when 1a was treated with $\mathrm{DMPU} / \mathrm{HCl}$ alone (Table 1, entries 1-3). Strong hydrogen-bond-donating solvents such as HFIP (hexafluoro-2-propanol) can form hydrogen bond networks or clusters, ${ }^{17}$ which have been shown to provide significant increases in the rate of many reactions. ${ }^{18}$ To our delight, by using a two-component solvent mixture of DCM/HFIP, an excellent yield of syn-addition product 2 a was obtained (Table 1, entry 4). Notably, lower reactivity and stereoselectivity were observed when commercial solutions of $\mathrm{HCl}$ were used (Table 1 , entries 5-7).

We then focused our attention on obtaining the antiaddition isomer using transition-metal catalysis. First, we evaluated several literature-known alkyne hydrochlorination catalytic systems, and we found that neither $\mathrm{TiO}_{2} / \mathrm{Au}^{12 \mathrm{e}}$ nor $\mathrm{CpRuCl}(\mathrm{cod}) / \mathrm{PPh}_{3}{ }^{12 \mathrm{~d}}$ gave good yields and stereoselectivity (Table 1, entries 8-9). Recently, we reported an efficient homogeneous gold-catalyzed hydrochlorination procedure, ${ }^{19}$ but when the same conditions were used in this case, 3a was obtained in low yields, although good stereoselectivity was observed (Table 1, entries 10-11). We reasoned that we could enhance the reactivity by adding hydrogen bond donor HFIP as a part of the solvent mixture, but this resulted in an $E / Z$ mixture (Table 1, entry 12 ), possibly due to competition of the
Brønsted-acid-catalyzed process (see Table 1, entry 4). This prompted us to turn to alternative chloride sources. To our satisfaction, a combination of $\mathrm{LiCl} / \mathrm{AcOH}$ with JohnPhosAuCl dramatically increased both chemical yield and anti-selectivity (Table 1, entry 13). We also investigated chlorination systems using in situ generated $\mathrm{HCl}$, but they were less effective (Table 1 , entries 14-15).

With the optimized conditions in hand, we first evaluated the hydrogen-bonding-assisted syn-hydrochlorinations (Table 2 ). We found that iodo-, bromo-, and chloroalkynes were all

Table 2. Substrate Scope of (E)-2-Halo-1-chloroalkenes

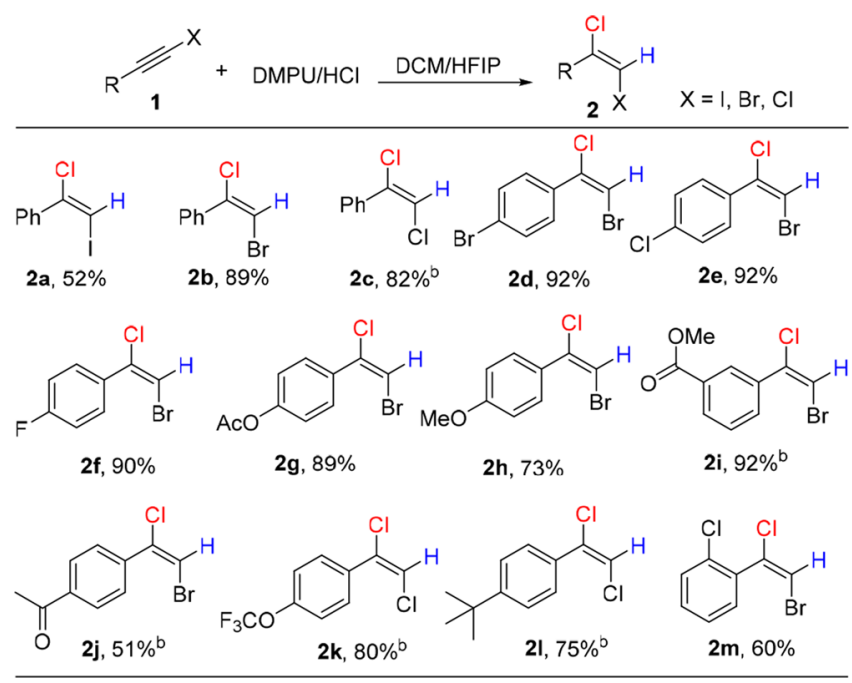

${ }^{a}$ Condition: haloalkyne $1(0.2 \mathrm{mmol}), \mathrm{DMPU} / \mathrm{HCI}(0.4 \mathrm{mmol})$, DCM/HFIP $(3: 1,0.4 \mathrm{~mL}), 50{ }^{\circ} \mathrm{C}$ for $8 \mathrm{~h} .{ }^{b}$ Reaction carried out at 70 ${ }^{\circ} \mathrm{C}$.

suitable substrates (Table 2, 2a-c).$^{20}$ Our conditions exhibited high compatibility with halogens $(\mathrm{F}, \mathrm{Cl}$, or $\mathrm{Br}$ ) (Table 2, 2df) and the acid-sensitive acetyl group (Table 2, 2g). Electronwithdrawing groups such as esters or ketones reduced the reactivity, and a higher temperature $\left(70{ }^{\circ} \mathrm{C}\right)$ was needed (Table 2, 2i,j). Furthermore, the trifluoromethoxy group and the tert-butyl group were also well-tolerated, achieving yields of $80 \%$ and $75 \%$, respectively (Table $2,2 \mathbf{k}, \mathbf{l}$ ). However, aliphatic haloalkynes showed no reactivity under the same conditions.

We next investigated the scope of the gold-catalyzed antihydrochlorination of bromoalkynes (Table 3, entries 3a-3w). Because of higher reactivity and milder conditions, this system offers outstanding functional group tolerance and excellent chemical yields aromatic alkynes substituted with halogens in both the ortho and para positions (Table 3, 3b-e); acidsensitive groups such as esters $(\mathbf{3} \mathbf{f}, \mathbf{3} \mathbf{j})$, nitriles $(\mathbf{3 h})$, and sulfonates (3k); ketones $(\mathbf{3 g})$, nitro groups (3i), and ethers $(3 \mathrm{~m})$ all worked very well. The aromatic diyne 31 was also shown to be a suitable substrate. In general, substrates with electron-withdrawing groups on the phenyl ring gave higher yields $(\mathbf{3} \mathbf{f}-\mathbf{i})$ than those with electron-donating groups such as $3 \mathrm{~m}$. Substrates containing heteroaromatics such as thiophene (3n) and pyridine (3o) also reacted efficiently, albeit with lower stereoselectivity. Contrary to the Brønsted acid-catalyzed process, we also found aliphatic haloalkynes to be suitable substrates with good to excellent yields $(3 \mathbf{p}-\mathbf{w})$ in the goldcatalyzed process. A variety of functional groups, including benzyl groups (3p), phthalimides (3q), alkenes (3t), alcohols $(3 \mathbf{u})$, and carboxylic acids (3v) were all well-tolerated. Even 
Table 3. Scope of Gold-Catalyzed Hydrochlorination of Haloalkynes

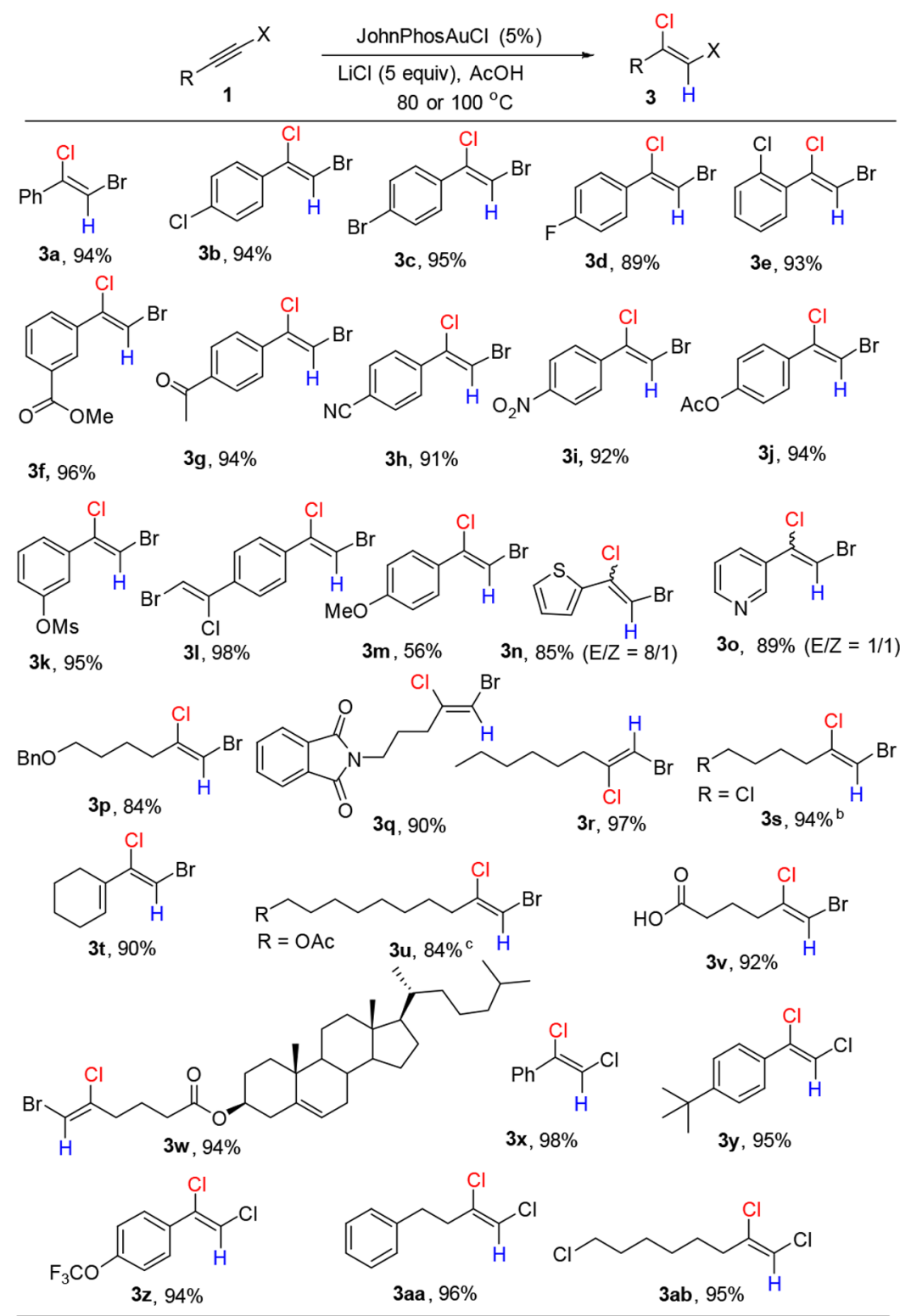

${ }^{a}$ Reaction conditions: haloalkynes $1(0.2 \mathrm{mmol}), \mathrm{LiCl}(1.0 \mathrm{mmol})$, JohnPhosAuCl $(5 \mathrm{~mol} \%)$, and $\mathrm{HOAc}(0.5 \mathrm{~mL}), 80^{\circ} \mathrm{C}$ (for bromoalkynes) or $100{ }^{\circ} \mathrm{C}$ (for chloroalkynes). ${ }^{b}$ Start from $1 \mathbf{s}(\mathrm{R}=-\mathrm{OTs}) .{ }^{c}$ Start from $1 \mathbf{u}(\mathrm{R}=-\mathrm{OH})$.

the cholesterol-tethered substrate $3 \mathbf{w}$ gave a high yield and selectivity. We also explored the substrate scope of chloroalkynes $(3 \mathbf{x}-\mathbf{3 a b})$. It should be noted that some functional groups such as $-\mathrm{OTs}$ and $-\mathrm{OH}$ were chlorinated or esterified $(3 \mathbf{s}, 3 \mathbf{u})$ under the reaction condition. While chloroalkynes are less reactive, thus requiring high temperatures $\left(100{ }^{\circ} \mathrm{C}\right)$, the excellent yields and exclusive antiselectivity were maintained. Various functional groups such as tert-butyl (3y), trifluoromethoxyl (3z), and chloroalkyl (3ab) were well-tolerated. We also tested reactions of iodoalkynes, but they did not give clean transformations.

The proposed mechanism is shown in Scheme 2. ${ }^{21}$ The hydrogen-bonding cluster generated from HFIP forms a hydrogen-bonding complex with $\mathrm{HCl}$ (A) and grants the $\mathrm{HCl}$ increased acidity, facilitating the rate-determining protontransfer step (Scheme 2a). This gives rise to intermediate vinyl cation $\mathbf{B},{ }^{22}$ which should have a linear geometry, ${ }^{23}$ which we confirmed with DFT calculations (Scheme 2a). Orbitals from the bromine atom participate in stabilizing the LUMO of $\mathbf{B}$. Attack at the upper face is hindered by the bulky bromine atom, thus favoring chloride attack syn to the $\mathrm{H}$ atom ${ }^{24}$

On the other hand, the gold-catalyzed process gave the expected anti-selectivity, consistent with typical homogeneous gold catalysis (Scheme $2 \mathrm{~b}$ ). ${ }^{25}$ Contrary to traditional silverbased cationic gold catalysis, we used hydrogen bonding network to activate cationic gold catalyst precursor ( $\mathrm{L}-\mathrm{Au}-$ $\mathrm{Cl}) .{ }^{26}$ We proposed that the $\mathrm{H}$-bond network generated from acetic acid might weaken the $\mathrm{Au}-\mathrm{Cl}$ bond, thereby generating a gold species $[\mathrm{Au}]$ with a partial positive charge that coordinated to the haloalkyne (Scheme 2b). The chloride nucleophile would then attack the gold-activated haloalkyne to generate a vinyl-gold intermediate $\mathbf{C}$ in an anti-manner. Subsequent protodeauration of $\mathbf{C}$ by $(\mathrm{AcOH})_{n}$ would therefore afford the anti-product. ${ }^{27}$ This is consistent with our deuterium-labeling experiment: using $d_{4}-\mathrm{AcOH}$ as the solvent, the deuterated product was obtained in $92 \%$ yield (eq 1 ). This 
Scheme 2. Proposed Mechanism ${ }^{a}$

a) Hydrogen bond network assisted acid catalysis (syn-addition)

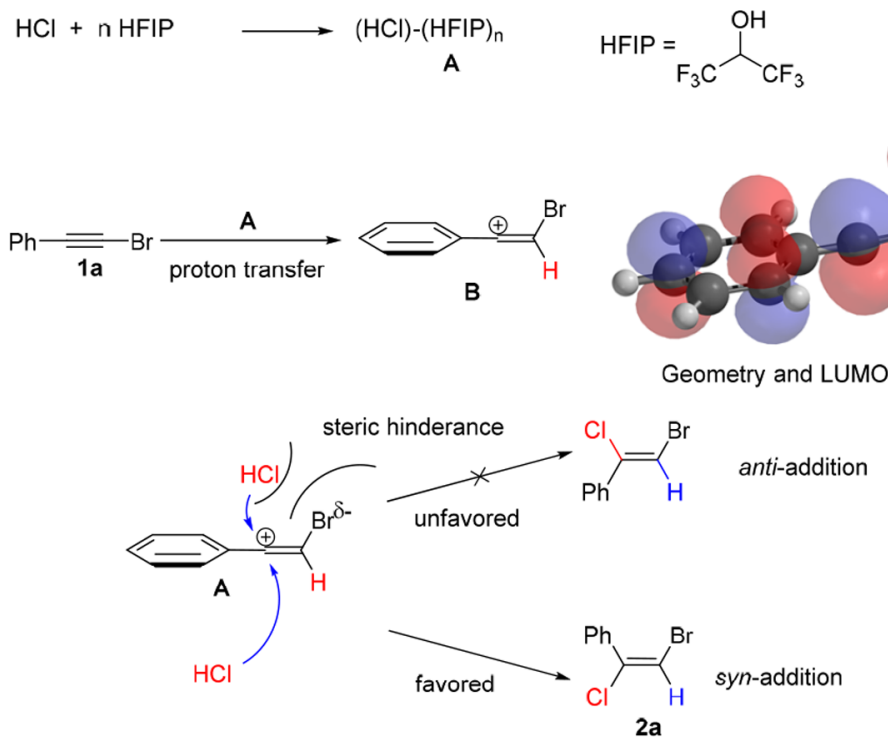

b) Hydrogen bond network assisted gold catalysis (anti-addition)
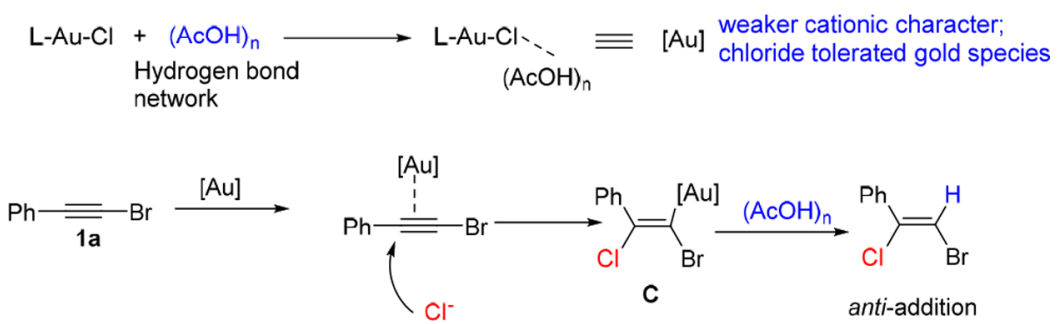

${ }^{a} \mathrm{LUMO}$ of vinyl cation was calculated at B3LYP/6-311+G(2df,2p) level of theory.

result indicates that $\mathrm{AcOH}$ is the proton source; hence, this method could be used to synthesize other deuterium labeled haloalkenes.

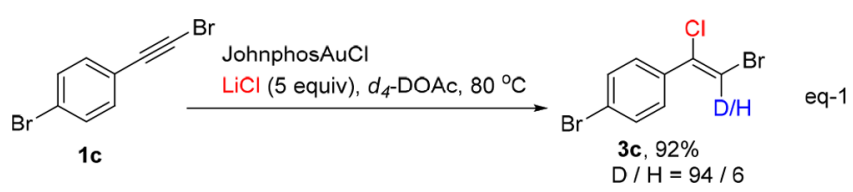

Our protocol is scalable: similarly good yields were obtained at a larger scale (Scheme 3a). Alkenyl chlorides have become increasingly suitable substrates in cross-coupling reactions due to the advances in catalyst design. ${ }^{28}$ Taking advantage of the difference in reactivity between chloro-and bromo/chloroalkenes, we were able to conduct sequential cross-coupling reactions to synthesize both $E$ - and $Z$-trisubstituted alkenes: Sonogashira $^{4 \mathrm{~b}}$ and Suzuki-Miyaura ${ }^{29}$ coupling reactions provided an efficient and selective way to make both $(Z)$ and $(E)$-enynes (Scheme $3 \mathrm{~b}, \mathrm{c}){ }^{14}$ What's more, our method represents a simple way of accessing chlorinated 1,3-dienes such as 3t (Table 3), which can be further transformed into multifunctional aromatics in good yields via tandem DielsAlder-aromatization. ${ }^{14}$

In summary, we have developed a widely applicable, highly efficient synthesis of both (Z)- and (E)-1,2-haloalkenes via hydrochlorination of haloalkynes. Other applications of
Scheme 3. Larger-Scale Reactions and Further Transformations of $(Z)$ - and (E)-1,2-Haloalkenes

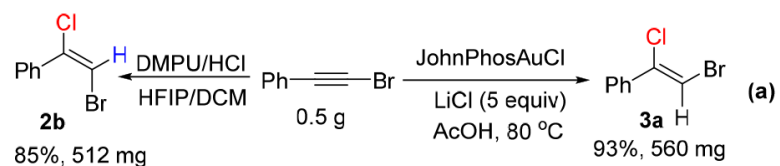

$85 \%, 512 \mathrm{mg}$
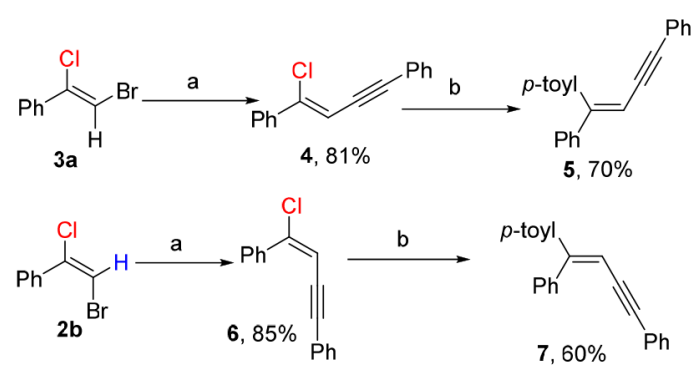

${ }^{a}$ Phenylacetylene (1.5 equiv), $\mathrm{Pd}\left(\mathrm{PPh}_{3}\right)_{2} \mathrm{Cl}_{2}$ (5 mol \%), $\mathrm{Cul}(15 \%)$, $\mathrm{Et}_{3} \mathrm{~N}$ (3.0 equiv), toluene, $80{ }^{\circ} \mathrm{C} .{ }^{b} \mathrm{Pd}(\mathrm{OAc})_{2}(5 \mathrm{~mol} \%), \mathrm{PPh}_{3}(10 \%)$, $p-\mathrm{CH}_{3}-\mathrm{C}_{6} \mathrm{H}_{4} \mathrm{~B}(\mathrm{OH})_{2}$ (1.5 equiv), $\mathrm{Cs}_{2} \mathrm{CO}_{3}$ (2.0 equiv), dioxane, 90 ${ }^{\circ} \mathrm{C}$.

hydrogen bonding network-assisted Brønsted acid and gold catalytic systems are currently being investigated in our laboratories. 


\section{ASSOCIATED CONTENT}

\section{S Supporting Information}

The Supporting Information is available free of charge on the ACS Publications website at DOI: 10.1021/acscatal.7b03563.

Experimental details and copies of NMR spectra (PDF)

\section{AUTHOR INFORMATION}

\section{Corresponding Authors}

*E-mail: bo.xu@dhu.edu.cn.

*E-mail: gb.hammond@louisville.edu.

ORCID $\odot$

Gerald B. Hammond: 0000-0002-9814-5536

Notes

The authors declare no competing financial interest.

\section{ACKNOWLEDGMENTS}

We are grateful to the National Science Foundation of China (NSFC-21472018). G.B.H. is grateful to the National Institutes of Health (R01GM121660). X.Z is grateful to the China Scholarship Council, and to Mr. Daniel M. Sedgwick for assisting in the preliminary editing of the manuscript.

\section{REFERENCES}

(1) (a) Wu, W.; Jiang, H. Acc. Chem. Res. 2014, 47, 2483-2504. (b) Johansson Seechurn, C. C. C.; Kitching, M. O.; Colacot, T. J.; Snieckus, V. Angew. Chem., Int. Ed. 2012, 51, 5062-5085.

(2) (a) Espinet, P.; Echavarren, A. M. Angew. Chem., Int. Ed. 2004, 43, 4704-4734. (b) Stille, J. K. Angew. Chem., Int. Ed. Engl. 1986, 25, $508-524$.

(3) Miyaura, N.; Suzuki, A. Chem. Rev. 1995, 95, 2457-2483.

(4) (a) Negishi, E.-i.; Anastasia, L. Chem. Rev. 2003, 103, 19792018. (b) Sonogashira, K.; Tohda, Y.; Hagihara, N. Tetrahedron Lett. 1975, 16, 4467-4470.

(5) (a) Hartwig, J. F. Acc. Chem. Res. 2008, 41, 1534-1544. (b) Ruiz-Castillo, P.; Buchwald, S. L. Chem. Rev. 2016, 116, 1256412649 .

(6) (a) Nunnery, J. K.; Engene, N.; Byrum, T.; Cao, Z.; Jabba, S. V.; Pereira, A. R.; Matainaho, T.; Murray, T. F.; Gerwick, W. H. J. Org. Chem. 2012, 77, 4198-4208. (b) Akiyama, T.; Takada, K.; Oikawa, T.; Matsuura, N.; Ise, Y.; Okada, S.; Matsunaga, S. Tetrahedron 2013, 69, 6560-6564. (c) Chung, W.-j.; Vanderwal, C. D. Angew. Chem., Int. Ed. 2016, 55, 4396-4434.

(7) (a) Nicolaou, K. C.; Bulger, P. G.; Sarlah, D. Angew. Chem., Int. Ed. 2005, 44, 4442-4489. (b) Jana, R.; Pathak, T. P.; Sigman, M. S. Chem. Rev. 2011, 111, 1417-1492. (c) Dounay, A. B.; Overman, L. E. Chem. Rev. 2003, 103, 2945-2964.

(8) (a) Yauchi, Y.; Ide, M.; Shiogai, R.; Chikugo, T.; Iwasawa, T. Eur. J. Org. Chem. 2015, 2015, 938-943. (b) Chen, D.; Chen, X.; Lu, Z.; Cai, H.; Shen, J.; Zhu, G. Adv. Synth. Catal. 2011, 353, 14741478. (c) Chen, X.; Kong, W.; Cai, H.; Kong, L.; Zhu, G. Chem. Commun. 2011, 47, 2164-2166. (d) Lemay, A. B.; Vulic, K. S.; Ogilvie, W. W. J. Org. Chem. 2006, 71, 3615-3618. (e) Ho, M. L.; Flynn, A. B.; Ogilvie, W. W. J. Org. Chem. 2007, 72, 977-983. (f) Ide, M.; Yauchi, Y.; Iwasawa, T. Eur. J. Org. Chem. 2014, 2014, 32623267. (g) Ide, M.; Yauchi, Y.; Shiogai, R.; Iwasawa, T. Tetrahedron 2014, 70, 8532-8538. (h) Chen, D.; Cao, Y.; Yuan, Z.; Cai, H.; Zheng, R.; Kong, L.; Zhu, G. J. Org. Chem. 2011, 76, 4071-4074. (i) Sproul, K. C.; Chalifoux, W. A. Org. Lett. 2015, 17, 3334-3337. (j) Li, J.; Yang, W.; Yang, S.; Huang, L.; Wu, W.; Sun, Y.; Jiang, H. Angew. Chem., Int. Ed. 2014, 53, 7219-7222. (k) Li, J.; Hu, W.; Li, C.; Yang, S.; Wu, W.; Jiang, H. Org. Chem. Front. 2017, 4, 373-376. (1) Blomquist, A. T.; Burge, R. E.; Sucsy, A. C. J. Am. Chem. Soc. 1952, 74, 3636-3642. (m) Wang, L.; Zhu, H.; Che, J.; Yang, Y.; Zhu, G. Tetrahedron Lett. 2014, 55, 1011-1013.
(9) Desrat, S.; Remeur, C.; Geny, C.; Riviere, G.; Colas, C.; Dumontet, V.; Birlirakis, N.; Iorga, B. I.; Roussi, F. Chem. Commun. 2014, 50, 8593-8596.

(10) Sun, C.; Camp, J. E.; Weinreb, S. M. Org. Lett. 2006, 8, 17791781.

(11) (a) Schuh, K.; Glorius, F. Synthesis 2007, 2007, 2297-2306. (b) Ochiai, M.; Uemura, K.; Oshima, K.; Masaki, Y.; Kunishima, M.; Tani, S. Tetrahedron Lett. 1991, 32, 4753-4756.

(12) (a) Weiss, H. M. J. Chem. Educ. 1995, 72, 848. (b) Podhajsky, S. M.; Sigman, M. S. Organometallics 2007, 26, 5680-5686. (c) Xu, C.-X.; Ma, C.-H.; Xiao, F.-R.; Chen, H.-W.; Dai, B. Chin. Chem. Lett. 2016, 27, 1683-1685. (d) Dérien, S.; Klein, H.; Bruneau, C. Angew. Chem., Int. Ed. 2015, 54, 12112-12115. (e) Oliver-Meseguer, J.; Doménech-Carbó, A.; Boronat, M.; Leyva-Pérez, A.; Corma, A. Angew. Chem., Int. Ed. 2017, 56, 6435-6439. (f) Wang, B.; Wang, S.; Li, P.; Wang, L. Chem. Commun. 2010, 46, 5891-5893. (g) Iwai, T.; Fujihara, T.; Terao, J.; Tsuji, Y. J. Am. Chem. Soc. 2012, 134, 12681274. (h) Schevenels, F. T.; Shen, M.; Snyder, S. A. J. Am. Chem. Soc. 2017, 139, 6329-6337. (i) Fahey, R. C.; Lee, D.-J. J. Am. Chem. Soc. 1968, 90, 2124-2131. (j) Griesbaum, K.; Rao, R.; Leifker, G. J. Org. Chem. 1982, 47, 4975-4981. (k) Carothers, W. H.; Coffman, D. D. J. Am. Chem. Soc. 1932, 54, 4071-4076. (1) Marcuzzi, F.; Melloni, G. J. Am. Chem. Soc. 1976, 98, 3295-3300. (m) Kropp, P. J.; Crawford, S. D. J. Org. Chem. 1994, 59, 3102-3112. (n) Wang, B.; Jiang, J.-1.; Yu, H.-z.; Fu, Y. Organometallics 2017, 36, 523-529. (o) Yu, W.; Jin, Z. J. Am. Chem. Soc. 2000, 122, 9840-9841. (p) Ohashi, K.; Mihara, S.; Sato, A. H.; Ide, M.; Iwasawa, T. Tetrahedron Lett. 2014, 55, 632635. (q) Derosa, J.; Cantu, A. L.; Boulous, M. N.; O’Duill, M. L.; Turnbull, J. L.; Liu, Z.; De La Torre, D. M.; Engle, K. M. J. Am. Chem. Soc. 2017, 139, 5183-5193. (r) Mulder, J. A.; Kurtz, K. C. M.; Hsung, R. P.; Coverdale, H.; Frederick, M. O.; Shen, L.; Zificsak, C. A. Org. Lett. 2003, 5, 1547-1550. (s) Bönnemann, H.; Korall, B. Angew. Chem., Int. Ed. Engl. 1992, 31, 1490-1492. (t) Lu, X.-Y.; Zhu, G.-X.; Ma, S.-M. Chin. J. Chem. 1993, 11, 267-271. (u) Li, W.; Gao, L.; Yue, Z.; Zhang, J. Adv. Synth. Catal. 2015, 357, 2651-2655.

(13) Chen, Z.; Jiang, H.; Li, Y.; Qi, C. Chem. Commun. 2010, 46, 8049-8051.

(14) Zhu, G.; Chen, D.; Wang, Y.; Zheng, R. Chem. Commun. 2012, $48,5796-5798$.

(15) (a) Hashmi, A. S. K.; Hutchings, G. J. Angew. Chem., Int. Ed. 2006, 45, 7896-7936. (b) Hashmi, A. S. K. Chem. Rev. 2007, 107, 3180-3211. (c) Hutchings, G. J. Top. Catal. 2008, 48, 55-59.

(16) Liang, S.; Ebule, R.; Hammond, G. B.; Xu, B. Org. Lett. 2017, $19,4524-4527$.

(17) Berkessel, A.; Adrio, J. A. J. Am. Chem. Soc. 2006, 128, 1341213420 .

(18) (a) Liu, W.; Wang, H.; Li, C.-J. Org. Lett. 2016, 18, 2184-2187. (b) Colomer, I.; Batchelor-McAuley, C.; Odell, B.; Donohoe, T. J.; Compton, R. G. J. Am. Chem. Soc. 2016, 138, 8855-8861. (c) Tian, Y.; Xu, X.; Zhang, L.; Qu, J. Org. Lett. 2016, 18, 268-271. (d) Zeng, X.; Liu, S.; Shi, Z.; Xu, B. Org. Lett. 2016, 18, 4770-4773.

(19) Ebule, R.; Liang, S.; Hammond, G. B.; Xu, B. ACS Catal. 2017, 7, 6798-6801.

(20) (a) Nösel, P.; Müller, V.; Mader, S.; Moghimi, S.; Rudolph, M.; Braun, I.; Rominger, F.; Hashmi, A. S. K. Adv. Synth. Catal. 2015, 357, 500-506. (b) Nösel, P.; Lauterbach, T.; Rudolph, M.; Rominger, F.; Hashmi, A. S. K. Chem. - Eur. J. 2013, 19, 8634-8641. (c) Weyrauch, J. P.; Hashmi, A. S. K.; Schuster, A.; Hengst, T.; Schetter, S.; Littmann, A.; Rudolph, M.; Hamzic, M.; Visus, J.; Rominger, F.; Frey, W.; Bats, J. W. Chem. - Eur. J. 2010, 16, 956-963.

(21) Hashmi, A. S. K. Angew. Chem., Int. Ed. 2010, 49, 5232-5241.

(22) Wurm, T.; Bucher, J.; Duckworth, S. B.; Rudolph, M.; Rominger, F.; Hashmi, A. S. K. Angew. Chem., Int. Ed. 2017, 56, 3364-3368.

(23) Stang, P. J.; Summerville, R. J. Am. Chem. Soc. 1969, 91, 46001 .

(24) Métayer, B.; Compain, G.; Jouvin, K.; Martin-Mingot, A.; Bachmann, C.; Marrot, J.; Evano, G.; Thibaudeau, S. J. Org. Chem. 2015, 80, 3397-3410. 
(25) Hashmi, A. S. K.; Weyrauch, J. P.; Frey, W.; Bats, J. W. Org. Lett. 2004, 6, 4391-4394.

(26) Ebule, R.; Liang, S.; Hammond, G. B.; Xu, B. ACS Catal. 2017, 7, 6798 .

(27) Nunes dos Santos Comprido, L.; Klein, J. E. M. N.; Knizia, G.; Kästner, J.; Hashmi, A. S. K. Chem. - Eur. J. 2017, 23, 10901-10905. (28) (a) Barluenga, J.; Fernandez, M. A.; Aznar, F.; Valdes, C. Chem. Commun. 2004, 1400-1401. (b) Dai, C.; Fu, G. C. J. Am. Chem. Soc. 2001, 123, 2719-2724. (c) Littke, A. F.; Dai, C.; Fu, G. C. J. Am. Chem. Soc. 2000, 122, 4020-4028. (d) Su, W.; Urgaonkar, S.; McLaughlin, P. A.; Verkade, J. G. J. Am. Chem. Soc. 2004, 126, 16433-16439. (e) Venkat Reddy, C. R.; Urgaonkar, S.; Verkade, J. G. Org. Lett. 2005, 7, 4427-4430. (f) Organ, M. G.; Ghasemi, H.; Valente, C. Tetrahedron 2004, 60, 9453-9461. (g) Cristau, H.-J.; Cellier, P. P.; Hamada, S.; Spindler, J.-F.; Taillefer, M. Org. Lett. 2004, 6, 913-916.

(29) Miyaura, N.; Yamada, K.; Suzuki, A. Tetrahedron Lett. 1979, 20, 3437-3440. 